\title{
The birth of strange stars and their dynamo-originated magnetic fields
}

\author{
R. X. $\mathrm{Xu}^{1,2}$ and F. H. Busse ${ }^{2}$ \\ 1 Beijing Astrophysical Center and Astronomy Department, Peking University, Beijing 100871, PR China \\ 2 Theoretical Physics IV, University of Bayreuth, 95440 Bayreuth, Germany \\ e-mail: Friedrich.Busse@uni-bayreuth.de
}

Received 5 October 2000 / Accepted 12 March 2001

\begin{abstract}
It is shown that protostrange stars (PSSs) can be convective and that there are two possible scenarios describing their turbulence. Besides the local turbulence on the scale which is less than the mean free path of neutrinos, large-scale $(\sim 1 \mathrm{~km})$ convection also may occur with properties that are similar to those of convection in protoneutron stars (PNSs). We thus suggest that strange stars can also create dynamo-originated magnetic fields during the deleptonization episode soon after a supernova explosion. Further detailed investigations are needed to see whether or not strange stars and neutron stars can be distinguished according to the differences in dynamo actions in strange quark matter and in neutron matter. The magnetic fields of strange stars and neutron stars may also behave very differently during the accretion-phase when the fields decay.
\end{abstract}

Key words. stars: pulsars: general - stars: neutron - elementary particles - stars: supernovae: general magnetic fields

\section{Introduction}

As ordinary nucleons (neutrons and protons), which are composed of quarks, are squeezed tightly enough at high temperature and pressure, they may turn into a soup of deconfined free quarks. This type of phase transition might be expected in the center of a neutron star which consists mainly of neutron matter. In the conventional picture of neutron stars with quark matter, the neutron (hadron) and quark phases are separated by a sharp boundary with density discontinuity; such stars are called "hybrid" stars. But, as first considered by Glendenning (1992) for "complex" systems, it is possible that bulk quark and nuclear matter could coexist over macroscopical distances in neutron stars; such stars are called "mixed" stars (see, e.g., Heiselberg et al. 2000 for a recent review about hybrid and mixed stars). A further radical view is that the whole neutron star should be phase-converted to be a strange star, which consists of nearly equal numbers of $u, d$, and $s$ quarks and associated electrons for charge neutrality, if strange quark matter (SQM) is the true ground state of strong interacting matter (Bodmer 1971; Witten 1984; see also, e.g., Madsen 1999, for a recent review of the

Send offprint requests to: R. X. Xu, e-mail: rxxu@bac.pku.edu.cn physics and astrophysics of strange matter and strange stars). Strange stars provide a sharp contrast to neutron stars (e.g. the mass-radius relations and the surface conditions), although both neutron and strange stars may have similar radii $(\sim 10 \mathrm{~km})$ and masses $\left(\sim 1.4 M_{\odot}\right)$.

Pulsars can be modeled as neutron stars or strange stars. No strong observational evidence known hitherto favors either of the models. It is thus of great importance and interest in current astrophysics to distinguish neutron stars and strange stars observationally. Recently, at least three hopeful ways have been proposed for identifying a strange star: 1, hot strange stars (or neutron stars containing significant quantities of strange matter) may rotate more rapidly since the higher bulk viscosity (Wang \& Lu 1984; Madsen 1992) of strange matter can effectively damp away the $r$-mode instability (Madsen 1998); 2, the approximate mass-radius $(M-R)$ relations of strange stars $\left(M \propto R^{3}\right)$ are in striking contrast to those of neutron stars $\left(M \propto R^{-3}\right)$. Comparisons of observationdetermined relations with theoretical relations may thus determine whether an object is a neutron star or a strange star ( $\mathrm{Li}$ et al. 1999); 3, It is possible to distinguish "bare" polar cap strange stars from neutron stars via pulsar magnetospheric and polar radiation because of the striking differences between the polar surface conditions of the two 
types of stars (e.g., clear drifting pattern from "antipulsars", Xu et al. 1999; Xu et al. 2001). In this paper, we shall study possible differences in the processes of magnetic field generation by dynamo action in strange stars and in neutron stars which could eventually lead to observational distinctions between the two types of stars.

Magnetic fields play a key role in pulsar life. Unfortunately, there is still no consensus on the physical origin of strong fields. Naively, it is supposed that pulsars' fields are the result of the conservation of magnetic flux during supernova collapses. This idea faces at least two problems (Thompson \& Duncan 1993, here after TD93): 1 , dynamo action can occur in the convective episodes of both the iron core and the newborn star; 2, very strong fields $\left(>10^{12} \mathrm{G}\right.$ ) of pulsars are probably not "fossil fields" since only a few percent of white dwarfs have fields in excess of $10^{6}$ G. Based on the Newtonian scalar virial theorem, one can estimate the limiting interior magnetic field $B_{\max } \sim 10^{18} M_{1} R_{6}^{-2} \mathrm{G}$ of a star with mass $M=M_{1} M_{\odot}$ and radius $R=R_{6} 10^{6} \mathrm{~cm}$ (Lai \& Shapiro 1991). Both the estimate based on flux conservation and that based on the virial argument, do not depend on the detailed fluid properties of the stellar interiors. Thompson \& Duncan (TD93, Duncan \& Thompson 1992) have extensively considered turbulent dynamo amplification in protoneutron stars (PNSs) and in the progenitor stars, and found that multipolar structure fields as strong as $10^{16} \mathrm{G}$ ("magnetar") can be generated by PNS convection. This suggestion seems to have been confirmed by recent observations (e.g., Kouveliotou et al. 1998). Furthermore, there arises a possible way to distinguish strange stars from neutron stars since the dynamo-originated fields depend on the detailed fluid properties of the stellar interiors. One interesting question thus is: Can strange stars create magnetic fields as strong as $10^{16} \mathrm{G}$ ? (i.e., can strange stars act as magnetars?). If strange stars can, is there any difference between the processes of field generation in PNSs and that in "proto-" strange stars (PSSs)? Theoretically, there is little work known hitherto on the generation of the magnetic fields of strange stars. It is supposed that spontaneous magnetization could result in the generation of a compact quark star (Tatsumi 2000). Alternatively, we suggest that dynamo-amplification of the magnetic field could also play an important role in PSSs with high temperatures. In this work, we are trying to find whether there are any differences between the dynamo-originated fields of neutron stars and that of strange stars, in order to contribute to the debate on the existence of strange stars. In our discussion, we presume that the strong magnetic fields of strange stars originate in the strange cores, rather than in the crusts with mass $\sim 10^{-5} M_{\odot}$, since strange stars produced during supernova explosions cannot have such crusts (Xu et al. 2001).

One difficult issue in the study of dynamo-originated fields of strange stars is to determine whether color superconductivity (CSC) occurs in PSS since the dynamo mechanism may not work effectively in a superconduct- ing plasma ${ }^{1}$. Recent calculations, based on a model where quarks interact via a point-like four-fermion interaction, showed that the energy gap $\Delta$ of zero-temperature strange matter could be 10-100 MeV for plausible values of the coupling (Alford et al. 1999); thus the critical temperature of forming possible quark Cooper pairs $T_{\mathrm{c}} \sim \Delta / 2 \sim$ $5-50 \mathrm{MeV}$. However, $T_{\mathrm{c}}$ could be altered if a more realistic quark-quark interaction is used and/or if the the trapping of neutrinos in PSSs is included. It is also hard to sufficiently determine the temperature $T$ (PSS) of PSSs. We therefore simply assume in this paper that $T(\mathrm{PSS})>T_{\mathrm{c}}$ in the first few seconds (the time-scale for dynamo action of PSS) after PSS formation.

\section{The birth of strange stars: differential rotation and convection?}

The time-scale of PNSs (or PSSs) is of the order of a few seconds, which is three orders of magnitude longer than the dynamical one. It is thus worth studying the dynamical bulk evolution in these stars, such as differential rotation and convection. Unfortunately, no numerical model of supernova explosion known hitherto has included the conversion from PNSs to PSSs, although such a conversion may help in modelling the burst process (e.g., 1, helping to solve the present energetic difficulties in getting type II supernova explosions (Benvenuto \& Horvath 1989); 2, giving a reasonable explanation for the second peak of neutrino emission in SN 1987A (Benvenuto \& Lugones 1999)). The absence of a numerical model is caused by the lack of (1) a full theory determining the conditions at which the quark matter phase transition occurs and of (2) a detailed understanding of the complex burning process of neutron matter into strange matter.

Nevertheless, some efforts have been made in trying to understand the transition and combustion processes in detail. The phase conversion occurs in two steps: first neutron matter deconfinement occurs on a strong interaction time scale $\sim 10^{-23} \mathrm{~s}$, then chemical equilibration of the deconfined quark matter takes place on a weak interaction time scale $\sim 10^{-8}$ s. Additional neutrinos and energy are produced in the second step (Dai et al. 1995). Further calculations of such transitions (Anand et al. 1997; Lugones \& Benvenuto 1998) have also considered the effect of strong interactions, the effect of finite temperature and strange quark mass, and the effect of trapped neutrinos. Recently, Benvenuto \& Lugones (1999) explored the occurrence of deconfinement transition in a PNS modeled by Keil \& Janka (1995) and found that the deconfinement appears as long as the bag constant $\check{B} \leq$ $126 \mathrm{MeV} \mathrm{fm}^{-3}$. Various estimates of the bag constant indicate that the preferred value of $\check{B}$ lies in the range of $60 \mathrm{MeV} \mathrm{fm}^{-3} \leq \check{B} \leq 110 \mathrm{MeV} \mathrm{fm}^{-3}$ (Drago 1999), which means that deconfinement is very likely to happen.

\footnotetext{
1 In case of CSC, the field creation and evolution may also be influenced significantly if there exist fast dynamos with magnetic Reynolds number $\mathcal{R}_{\mathrm{m}} \rightarrow \infty$ in PSSs.
} 
Such 2-flavor quark matter may be transformed immediately into a 3 -flavor one if SQM is absolutely stable. From a kinetic point of view, Olinto (1987) has calculated for the first time the conversion of neutron stars into strange stars, suggesting a deflagration mode with a burning velocity range from $10^{4} \mathrm{~km} \mathrm{~s}^{-1}$ to a few $\mathrm{cm} / \mathrm{s}$. However it is found (Horvath \& Benvenuto 1988; Benvenuto et al. 1989; Benvenuto \& Horvath 1989) that such slow modes are unstable. This instability would be self-accelerated, and the burning should occur finally in detonation modes, although the transition from deflagration to detonation has not been well understood (Lugones et al. 1994). In order for the combustion to be exothermic, there exists a minimum density $\rho_{\mathrm{c}}$ for detonation to be possible, $\rho_{\mathrm{c}} \sim 2$ times of nuclear density for some cases (Benvenuto \& Horvath 1989). Thus the detonation flame can not reach the edge of the compact core, and the outer part of the PNS would be expelled. As a result, a strange star is formed with an almost "bare" quark surface which is essential to solve completely the "binding energy" problem in some current pulsar emission models (Xu et al. 1999, 2001). Both the extra neutrino emissivity and the detonation wave can favour a successful core-collapse supernova explosion.

\subsection{Differential rotation}

It is expected that PNSs (thus PSSs) have a strong differential rotation (e.g., Janka \& Mönchmeyer 1989; Goussard et al. 1998). This issue is uncertain, however, due to the lack of a rotating core model in the pre-supernova evolution simulations. The iron core collapses, being triggered by electron capture (for lower entropy core) and/or photodisintegration (for higher entropy core), almost in the same way as for free fall. Many initial core models of highly evolved massive stars without rotation have appeared in the literature (e.g., Arnett 1977; Bruenn 1985), in which the density $\rho_{0}-$ radius $r_{0}$ relation can be fitted by

$\rho_{0}\left(r_{0}\right)=\sum_{i=1}^{n} \rho_{0 i} \mathrm{e}^{-\alpha_{i} r_{0}}$,

where $\rho_{0 i}, \alpha_{i}$ are constants. For the first order approximation, we let $n=1$ in our following discussion. In this case, the Lagrangian mass coordinate $\mathcal{M}\left(r_{0}\right)$ is

$\mathcal{M}\left(r_{0}\right)=\frac{4 \pi \rho_{01}}{\alpha_{1}^{3}}\left[2-\left(2+2 \alpha_{1} r_{0}+\alpha_{1}^{2} r_{0}^{2}\right) \mathrm{e}^{-\alpha_{1} r_{0}}\right]$.

Setting $\mathcal{M}\left(10^{8}\right) \sim 1.4 M_{\odot}$ and $\rho_{0}\left(10^{8}\right) \sim 10^{8} \mathrm{~g} \mathrm{~cm}^{-3}$ for the core with radius $R_{0}=10^{8} \mathrm{~cm}$, one obtains ${ }^{2}$

$\overline{2}$ The density model $\rho_{0}\left(r_{0}\right)=\rho_{01} \mathrm{e}^{-\alpha_{1} r_{0}}$ with $\rho_{01}=$ $210^{10} \mathrm{~g} \mathrm{~cm}^{-3}, \alpha_{1}=5.410^{-8} \mathrm{~cm}^{-1}$, based on which we will calculate the nature of differential rotation of PSSs (or PNSs), represents well the initial core model of Bruenn (1985, Fig. 1, there $R_{0} \sim 10^{8} \mathrm{~cm}, \mathcal{M}\left(10^{8}\right) \sim 1.4 M_{\odot}, \rho_{0}(0)=210^{10} \mathrm{~g} \mathrm{~cm}^{-3}$, and $\left.\rho_{0}\left(10^{8}\right)=810^{7} \mathrm{~g} \mathrm{~cm}^{-3}\right)$. We thus think that the computation according to this simplified density model can give the correct order of magnitude result for the actual collapsed rotating core. $\rho_{01}=210^{10} \mathrm{~g} \mathrm{~cm}^{-3}, \alpha_{1}=5.410^{-8} \mathrm{~cm}^{-1}$, by Eq. (1) and Eq. (2). We assume that the core before collapse with a uniform rotation of period $P_{0}$ can also be approximated by Eq. (1) for lack of a rotation core model in simulations, and we investigate below the collapse of the iron core with the above parameters. Here we also assume that pulsar rotation is mainly the result of angular momentum conservation during the core collapse process, rather than of the kick at birth (Spruit \& Phinney 1998) ${ }^{3}$.

Based on the approximation, the total rotational energy $E_{0}$ of the core is

$E_{0}=\frac{16 \pi^{3} \rho_{01}}{3 P_{0}^{2}} \cdot \frac{\Im\left(\alpha_{1}, R_{0}\right)}{\alpha_{1}^{5}}$,

and the total angular momentum $M_{0}$ is

$M_{0}=\frac{16 \pi^{2} \rho_{01}}{3 P_{0}} \cdot \frac{\Im\left(\alpha_{1}, R_{0}\right)}{\alpha_{1}^{5}}$,

where the function $\Im(\alpha, R)$ is defined by

$$
\begin{aligned}
\Im(\alpha, R)= & 24-\left(24+24 \alpha R+12 \alpha^{2} R^{2}\right. \\
& \left.+4 \alpha^{3} R^{3}+\alpha^{4} R^{4}\right) \mathrm{e}^{-\alpha R} .
\end{aligned}
$$

It is found that $\lim _{\alpha \rightarrow 0} \Im(\alpha, R) / \alpha^{5}=R^{5} / 5$. Contrary to the case of the core before collapse, newborn strange stars may be well approximated as objects with homogeneous density (Alcock et al. 1986). For a strange star with mass $M=1.4 M_{\odot}$ and radius $R=10^{6} \mathrm{~cm}$, we find the density $\rho \sim 710^{14} \mathrm{~g} \mathrm{~cm}^{-3}$. During the adiabatic collapse process, in which the entropy of each mass element does not change significantly, toroidal forces are negligible (although a poloidal force can cause a shock wave). The angular momentum of each mass element is thus conserved, and PSSs should be in differential rotation. We assume that the material at a shell with radius $r_{0}$ of the iron core before collapse contracts to another shell with radius $r$ in a PSS,

$r^{3}=\frac{3}{4 \pi \rho} \mathcal{M}\left(r_{0}\right)$

According to angular momentum conservation of each mass element, one arrives at the velocity field $V$ dependent on $r$ and $\theta$ (polar angle),

$$
V=\frac{2 \pi \sin \theta}{P_{0}} \cdot \frac{r_{0}^{2}}{r}
$$

where $r_{0}$ is a function of $r$ through Eq. (6), and the mass conservation law $4 \pi r_{0}^{2} \rho_{0} \mathrm{~d} r_{0}=4 \pi r^{2} \rho \mathrm{d} r$ has been used. Owing to the momentum transport by neutrinos, magnetic fields, and turbulence, a uniform rotation is approached after a certain time and the final rotational energy $E_{\mathrm{f}}$ can be estimated as

$E_{\mathrm{f}}=\frac{16 \pi^{3}}{15 P^{2}} \rho R^{5} \sim 2.310^{46} P^{-2}$

${ }^{3}$ The recent observation of spin-kick alignment of Crab and Vela pulsars supports this suggestion. 


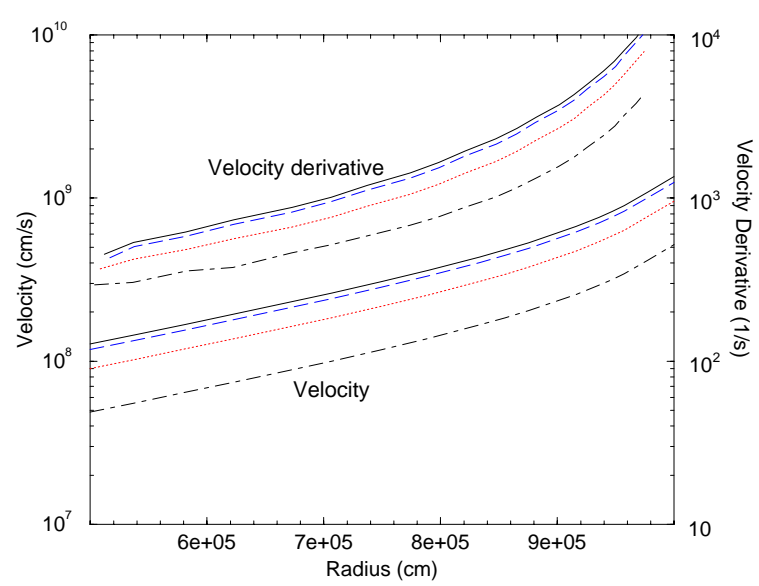

Fig. 1. The calculated velocity and velocity derivative profiles for different polar angles $\theta$. Dot-dashed, dotted, dashed, and solid lines are for $\theta=\pi / 8, \pi / 4,3 \pi / 8, \pi / 2$, respectively. The lower (upper) 4 lines, scaled by the left (right) ordinate, are for velocity (velocity derivative). $P=10 \mathrm{~ms}$ in the calculations

corresponding to the angular momentum $M_{\mathrm{f}}$,

$M_{\mathrm{f}}=\frac{16 \pi^{2}}{15 P} \rho R^{5}$,

where $P$ is the period of newborn uniformly rotating strange star. According to angular momentum conservation in the collapse process, $M_{0}=M_{\mathrm{f}}$, the rotation period $P_{0}$ of the initial iron core before collapse is

$P_{0}=\frac{5 \rho_{01} \Im\left(\alpha_{1}, R_{0}\right)}{\rho R^{5} \alpha_{1}^{5}} P \sim 4.6810^{3} P$,

for typical parameters, i.e., the core rotates with a period of about $50 \mathrm{~s}$ for an initial pulsar period $P \sim 10 \mathrm{~ms}$. From Eq. (7), one obtains the velocity derivative $|\nabla V|$,

$|\nabla V|=\sqrt{\left(\frac{\partial V}{\partial r}\right)^{2}+\left(\frac{1}{r} \frac{\partial V}{\partial \theta}\right)^{2}}$,

as a function of $\theta$ and $r$.

Figure 1 shows the velocity and the velocity derivative of this differential rotation scenario for $P=10 \mathrm{~ms}$, respectively, based on Eq. (7) and Eq. (11). We find $|\nabla V| \sim 10^{4} \mathrm{~s}^{-1}$ in the outer part of PNSs or PSSs. Such differential rotation may play an important role in the creation of magnetic fields of PSS or PNS, as will be discussed in Sect. 3.

Since the dynamo actions are in the outer convective layer with thickness $L \sim l_{\mathrm{p}} \sim 10^{5} \mathrm{~cm}$ (see Eq. (12) and Sect. 3), we give the velocity difference of differential rotation, $\Delta V=V\left(r=10^{6} \mathrm{~cm}\right)-V\left(r=910^{5} \mathrm{~cm}\right)$, as a function of $P$ in Fig. 2.

\subsection{Convection}

Turbulent convection in PNSs has been extensively investigated before (e.g. Burrows \& Lattimer 1988; Wilson \& Mayle 1988; Miralles et al. 2000). In the Kelvin-Helmholtz cooling phase, both the negative gradient of the entropy

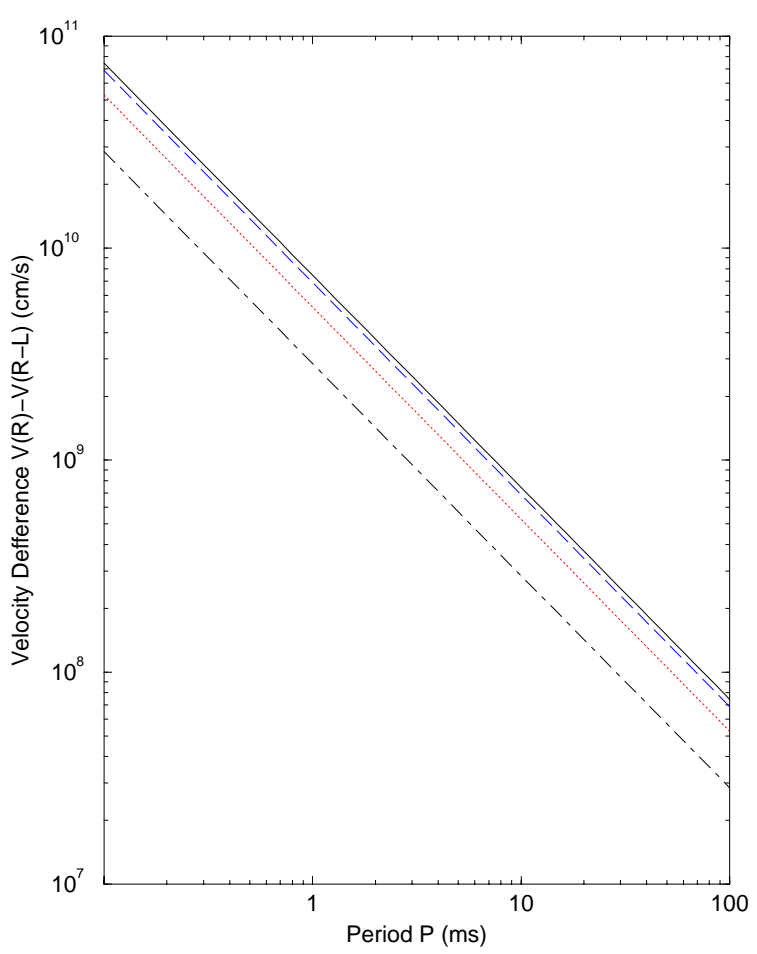

Fig. 2. The velocity difference in the convective outer layer with thickness of $L \sim 1 \mathrm{~km}$ as a function of pulsar initial period $P$ for different polar angles $\theta$. See Fig. 1 for the definition of lines

and of the lepton fraction can drive convection in newborn neutron stars, given that the mean free paths of leptons are much smaller than the convection length scale. Protostrange stars form after the SQM phase-transition with a time scale of $\sim R / c \sim 10^{-4} \mathrm{~s}$, which is much shorter than that of neutrino diffusion and thermal evolution, assuming that the actual combustion mode is detonation (Benvenuto et al. 1989).

We expect that PSSs are convective, since negative gradients of entropy and neutrino fractions would also appear in the outermost layers which can lose entropy and neutrinos faster than the inner part. Following arguments used by Thompson \& Duncan (TD93) in the case of PNSs, we compare the radiative and adiabatic temperature profiles to see whether convection occurs in PSSs. We assume neutrinos in the outer part of PSS are nondegenerate since there the neutrinos are lost rapidly. Iwamoto (1982) has shown that for the mean free path $l$ of the scattering of nondegenerate neutrinos by relativistic degenerate quark matter with temperature $T, 1 / l \propto T^{3}$ holds. Thus, the neutrino opacity in SQM scales as $T^{3}$ (rather than $T^{2}$ as for the case of PNSs), and the radiative temperature profile in PSSs is much steeper, $T(r) \propto p(r)$. We can employ the analytical equation of state for SQM in the case of zero strange quark mass $\left(m_{\mathrm{s}}=0\right)$ and zero coupling constant $\left(\alpha_{\mathrm{s}}=0\right)$ of strong interaction (Cleymans et al. 1986; Benvenuto et al. 1989) to estimate the adiabatic temperature-pressure relation. The entropy per baryon is $S \sim 3 \pi^{2} T / \mu$ for the case of chemical potential $\mu \sim 300 \mathrm{MeV}$ and temperature $T \sim 30 \mathrm{MeV}$. 
Therefore $T(r) \sim(p(r)+\check{B})^{1 / 4}$ for SQM moving with fixed $S$ is much less steep, and we can thus expect that a negative entropy gradient appears in the outer layer which is unstable to convection, similar to the case of PNSs. Accordingly we suggest that Schwarzchild convection exists in PSSs, whereas it will depend on detailed simulations whether Ledoux convection in PSSs can be established. If the timescale $\tau_{\mathrm{NM}}$ for neutron matter convection is much smaller than the neutrino diffusion time (a few seconds), we may expect that Ledoux convection takes place in a PSS since the negative gradient of the lepton fraction is nearly the same for PSSs as that for PNSs.

In the following, we try to estimate the properties of convection in PSSs. The local pressure scale height $l_{\mathrm{p}}$ in PSS is

$l_{\mathrm{p}}=\frac{p}{\rho g} \sim 210^{5} \mathrm{~cm}$,

where $g \sim \frac{G M}{R^{2}}=1.3310^{14} M_{1} R_{6}^{-2} \mathrm{~cm} \mathrm{~s}^{-2}\left(M_{1}=M / M_{\odot}\right.$, $R_{6}$ is the radius $R$ in $\left.10^{6} \mathrm{~cm}\right)$ is the gravitational acceleration, $p=\left(\rho c^{2}-4 \check{B}\right) / 3 \sim 10^{34}$ dyne $\mathrm{cm}^{-2}$ is the typical pressure in the outer convective layer, and the typical density there being chosen as $\rho=510^{14} \mathrm{~g} \mathrm{~cm}^{-3}$ (Alcock et al. 1986). We can assume the thickness $L$ of the outer layer where dynamo action exists to be equal to this scale height, $L \sim l_{\mathrm{p}}$, and the temperature gradient $\nabla T$ thus is

$\nabla T \simeq \frac{T}{L} \sim 510^{5} T_{11} \mathrm{~K} / \mathrm{cm}$,

where $T_{11}$ is the SQM temperature in $10^{11} \mathrm{~K}$. The numerical equation of state of SQM including the effect of nonzero strange quark mass (Eq. (29) of Chamaj \& Slominski 1989) is

$$
\begin{aligned}
\rho= & 1.0710^{14} \check{B}_{60}+3 \check{P} / c^{2}+1.0210^{-9} \check{P}^{0.6455} \\
& +1.3410^{-14} T^{2} \check{P}^{0.09619},
\end{aligned}
$$

where $\check{P} / c^{2} \equiv p / c^{2}+1.0710^{14} \check{B}_{60}\left(\check{B}_{60}\right.$ is the MIT bag constant in $60 \mathrm{MeV} \mathrm{fm}{ }^{-3}, p$ is the external pressure). According to Eq. (14) the coefficient of thermal expansion $\alpha$ is

$\alpha=-\frac{1}{\rho}\left(\frac{\partial \rho}{\partial T}\right)=2.6810^{-18} T_{11} \check{P}^{0.09619} \rho_{15}^{-1} \mathrm{~K}^{-1}$.

$\alpha=1.3310^{-14} \mathrm{~K}^{-1}$ for $T=10^{11} \mathrm{~K}$ and $p=0$ (Usov 1998).

There are two factors which can cause viscous stresses in a PSS: neutrino transport and quark scattering. Neutrino-induced viscosity dominates in a PSS on scales that are large compared to the neutrino mean-free path $l$. We use the neutrino mean-free path of nondegenerate neutrino scattering in SQM for a dimensional estimate (Iwamoto 1982),

$l=1.710^{2} \rho_{15}^{-2 / 3} E_{100}^{-3} \mathrm{~cm}$,

where $\rho_{15}$ is SQM density in $10^{15} \mathrm{~g} \mathrm{~cm}^{-3}$ and $E_{100}$ is neutrino energy in $100 \mathrm{MeV}$. According to Eq. (11) of TD93, the neutrino mean free path in nuclear matter,
$l^{\mathrm{N}} \sim 10^{2} \rho_{15}^{-1 / 3} \mathrm{~cm}$ for $T=30 \mathrm{MeV}$, which is of the same order as $l$. Unfortunately, no well-determined neutrino viscosity in PSS has appeared in the literature. We thus just estimate the neutrino-induced viscosity by a simple kinetic argument (Wilson \& Mayle 1988)

$\nu=\frac{1}{3} l c \xi=1.710^{10} \rho_{15}^{-2 / 3} E_{100}^{-3} \mathrm{~cm}^{2} \mathrm{~s}^{-1}$

where we have assumed the ratio $\xi$ of the neutrino energy density to the quark one to be $\sim 10^{-2}$. However, the kinematic viscosity due to quark scattering in SQM (Heiselberg \& Pethick 1993) is much smaller

$\nu_{\mathrm{q}} \sim 0.1 \rho_{15}^{14 / 9}\left(\alpha_{\mathrm{s}} / 0.1\right)^{-5 / 3} T_{11}^{-5 / 3} \mathrm{~cm}^{2} \mathrm{~s}^{-1}$.

Therefore turbulent convection may have a scale $\lesssim l$, which finally should be damped on a small scale by $\nu_{\mathrm{q}}$.

Two possibilities arise for the scenario of turbulence in PSSs. The first one is that the neutrino viscosity can effectively inhibit a large-scale convection with length scale $L$, and local convection with scale $\lesssim l$ will exist. In this case the local thermal diffusivity due to quark scattering in SQM (Heiselberg \& Pethick 1993) is

$\kappa_{\mathrm{q}} \sim 0.39 \rho_{15}^{2 / 3}\left(\alpha_{\mathrm{s}} / 0.1\right)^{-1} T_{11}^{-1} \mathrm{~cm}^{2} \mathrm{~s}^{-1}$.

The Prandtl number is

$P_{\mathrm{rq}}=\frac{\nu_{\mathrm{q}}}{\kappa_{\mathrm{q}}} \sim 0.25$,

the Rayleigh number is

$R_{\mathrm{aq}}=\frac{\alpha g \nabla T l^{4}}{\kappa_{\mathrm{q}} \nu_{\mathrm{q}}} \sim 7.210^{16}$

and the Coriolis number is

$\tau_{\mathrm{q}}=\frac{2 \Omega l^{2}}{\nu_{\mathrm{q}}} \sim 210^{8}$.

Malkus (1954) had estimated the mean-square values $\left(v^{2}\right)$ of the fluctuating velocity from the Boussinesq form of the hydrodynamic equations, and found (Eq. (64) in Malkus 1954)

$v \sim \frac{\kappa}{3 d}\left(R_{\mathrm{a}}-R_{\mathrm{ac}}\right)^{1 / 2}$,

where $\kappa$ is the thermal diffusivity, $d$ is the length scale of convection, $R_{\mathrm{a}}$ is the Rayleigh number, and $R_{\mathrm{ac}}$ is the critical Rayleigh number. This relation was supported by experiments (Fig. 2 in Malkus 1954) for $R_{\mathrm{a}}=10^{5}-10^{9}$. Also, Clever \& Busse (1981) found the perturbation energy $\left(v^{2}\right)$ increases nearly proportional to $R_{\mathrm{a}}-R_{\mathrm{ac}}$ at values of $R_{\mathrm{a}}>10^{3}$ for low-Prandtl-number convection $\left(P_{\mathrm{r}} \gtrsim 0.001\right.$, see Fig. 11 in Clever \& Busse 1981). According to Eq. (23), the turbulent convective velocity on this scale is

$v_{\mathrm{q}} \sim 3.510^{5} \mathrm{~cm} \mathrm{~s}^{-1}$

if we choose $\kappa=\kappa_{\mathrm{q}}, d=l, R_{\mathrm{a}}=R_{\mathrm{aq}}$.

The second scenario is that the neutrino viscosity is not high enough to inhibit the large-scale convection, and 
convection with scale $L$ is possible. We can also obtain some dimensionless numbers for this case. Since both the thermal energy and momentum in PSSs are transported by neutrinos now, the thermal diffusivity $\kappa$ can be estimated to be (Wilson \& Mayle 1988)

$\kappa=\frac{1}{3} l c=1.710^{12} \rho_{15}^{-2 / 3} E_{100}^{-3} \mathrm{~cm}^{2} \mathrm{~s}^{-1}$,

by a simple kinetic argument. Thus the Prandtl number is $P_{\mathrm{r}}=\frac{\nu}{\kappa}=\xi \sim 0.1-0.01$.

For a rotating spherical fluid shell with thickness $L$ and angular velocity $\Omega$, the Rayleigh number is

$R_{\mathrm{a}}=\frac{\alpha g \nabla T L^{4}}{\kappa \nu} \sim 6.010^{-23} T L^{3}$.

i.e. $R_{\mathrm{a}} \sim 6.010^{3}$ for $T \sim 10^{11} \mathrm{~K}, p=0$ and $L \sim 10^{5} \mathrm{~cm}$. The Taylor number $\tau^{2}$ can be calculated by

$\tau=\frac{2 \Omega L^{2}}{\nu}=1.210^{-10} \rho_{15}^{2 / 3} E_{100}^{3} \Omega L^{2}$.

$\tau \sim 1.210^{3}$ for $\Omega \sim 10^{3} \mathrm{~s}^{-1}$ and $L \sim 10^{5} \mathrm{~cm}$. The critical Rayleigh number $R_{\text {ac }}$ can be estimated (Zhang 1995) by

$R_{\mathrm{ac}} \simeq \sqrt{5 \tau} 10^{2} \sim 10^{4}$.

Turbulent convection is thus possible for $R_{\mathrm{a}}>R_{\mathrm{ac}}$ with a velocity which can be estimated to be $(d=L)$

$v \lesssim 410^{8} \mathrm{~cm} \mathrm{~s}^{-1}$,

according to Eq. (23). Alternatively, if the mixing-length prescription (Böhm-Vitense 1958, TD92) for a fluid of semidegenerate fermions is adapted, the convective velocity in PSS becomes

$v_{\mathrm{ml}}=\left(\frac{\Gamma-1}{2 \Gamma} \frac{L_{52}}{4 \pi R^{2} \rho}\right)^{1 / 3} \sim 6.810^{7} L_{52}^{1 / 3} \rho_{15}^{-1 / 3} \mathrm{~cm} \mathrm{~s}^{-1}$,

where $\Gamma \equiv \frac{\partial \ln p}{\partial \ln \rho}=\frac{\rho c^{2}}{\rho c^{2}-4 B} \sim 5, L_{52}$ is the convective luminosity in $10^{52} \mathrm{erg} \mathrm{s}^{-1}$. We note that $v_{\mathrm{ml}}$ and $v$ are nearly of the same order, while for PNSs with $\Gamma=5 / 3$, the convective velocity is $5.410^{7} L_{52}^{1 / 3} \rho_{15}^{-1 / 3} \mathrm{~cm} \mathrm{~s}^{-1} \sim 10^{8} \mathrm{~cm} \mathrm{~s}^{-1}$ for $\rho \sim 510^{14} \mathrm{~g} \mathrm{~cm}^{-3}$. Thus both, the convection in PSSs and in PNSs have convective velocities of about $10^{8} \mathrm{~cm} \mathrm{~s}^{-1}$ if large-scale convection is possible. Also the overturn timescales of PSSs and of PNSs are of the same order, $\tau_{\text {con }}=l_{\mathrm{p}} / v \sim$ a few ms, and the Rossby number is $R_{\mathrm{o}}=P / \tau_{\text {con }} \sim 10^{3} P_{1}$ in this possibility ( $P_{1}$ is the value of the initial period in seconds).

Another cause of turbulent motion in PSS (and PNS) is differential rotation. The Reynolds number $\mathcal{R}$ of this shear flow is

$\mathcal{R}=\frac{\nabla \check{U} \check{L}^{2}}{\nu}$,

where $\nabla \check{U}$ and $\check{L}$ are typical velocity derivative and length scales, respectively. For the local convection with scale $\lesssim l$, $\mathcal{R}_{\mathrm{q}} \sim 10^{8}$. For the convection with scale $L, \mathcal{R} \sim 5.810^{3}$. According to the experiment by van Atta (1966, Fig. 3 there), turbulence requires Reynolds number $\mathcal{R}>10^{4}$, thus in our case small scale $(\lesssim l)$ turbulence may appear owing to the differential rotation.

\section{Dynamo actions in proto-strange stars}

Magnetic fields in a static plasma of finite electric conductivity $\sigma$ are subject to diffusion and dissipation, the timescale of which is $\tau_{\mathrm{ohm}}=\check{L}^{2} / \kappa_{\mathrm{m}}=4 \pi \sigma \check{L}^{2} / c^{2}\left(\kappa_{\mathrm{m}}=\right.$ $c^{2} /(4 \pi \sigma)$ is the magnetic diffusivity). However, in a flowing plasma, magnetic fields may be created with certain velocity fields. This is the so-called "dynamo" process, which converts kinetic energy into magnetic energy (e.g., Moffatt 1978). The equation describing the time-variation of the magnetic field $\boldsymbol{B}$ governs the dynamo action

$\frac{\partial \boldsymbol{B}}{\partial t}=\nabla \times(\boldsymbol{v} \times \boldsymbol{B})+\mathcal{R}_{\mathrm{m}}^{-1} \nabla^{2} \boldsymbol{B}$,

where $\mathcal{R}_{\mathrm{m}}=\tau_{\mathrm{ohm}} / \tau_{\mathrm{v}}=\check{U} \check{L} / \kappa_{\mathrm{m}}$ is the magnetic Reynolds number $\left(\tau_{\mathrm{v}}=\check{L} / \check{U}\right.$ is the advection timescale). The magnetic diffusivity can be estimated

$\kappa_{\mathrm{m}}=\frac{c^{2}}{4 \pi \sigma}=5.810^{-3} \rho_{15}^{-8 / 9}\left(\alpha_{\mathrm{s}} / 0.1\right)^{5 / 3} T_{11}^{5 / 3} \mathrm{~cm}^{2} \mathrm{~s}^{-1}(34)$

for SQM (here $\sigma$ is calculated from Eq. (39) and Eq. (28) of Heiselberg \& Pethick 1993). Thus $\mathcal{R}_{\mathrm{m}} \sim 10^{16}$ for PSSs. But for PNSs, the magnetic diffusivity is (TD93)

$\kappa_{\mathrm{m}}^{\mathrm{N}}=1.510^{-5} \rho_{15}^{-1 / 3} \mathrm{~cm}^{2} \mathrm{~s}^{-1}$,

for neutron matter with an electron fraction of 0.2 , which is about two orders smaller than $\kappa_{\mathrm{m}}$. From Eq. (17) and Eq. (34), one obtains the magnetic Prandtl number $P_{\mathrm{m}}$

$P_{\mathrm{m}}=\frac{\nu}{\kappa_{\mathrm{m}}}=3.010^{12} \rho_{15}^{2 / 9} E_{100}^{-3}\left(\alpha_{\mathrm{s}} / 0.1\right)^{-5 / 3} T_{11}^{-5 / 3}$.

\subsection{An estimate of field strength}

Thompson \& Duncan (TD93) concluded that the dominant kinetic energy to be converted into magnetic energy in the dynamo action of PNSs is the convective energy since the initial pulsar rotation period is probably much larger than the overturn time $(\sim 1 \mathrm{~ms})$ of a convective cell, although large-scale $\alpha-\Omega$ dynamo action is essential for neutron stars with $R_{\mathrm{o}} \lesssim 1$ (i.e., initial period $\lesssim 1 \mathrm{~ms}$ ) to produce very high fields ("magnetars", Duncan \& Thompson 1992). However, here we suggest that rotation can not be neglected even for pulsars with typical initial periods, because the differential rotation energy density $\frac{E_{\mathrm{d}}-E_{\mathrm{f}}}{R^{3}} \sim 810^{31} \mathrm{erg} \mathrm{cm}^{-3}$ is even larger than the turbulent energy density $\rho v^{2} / 2 \sim 510^{30} \mathrm{erg} \mathrm{cm}^{-3}$ for $P=10 \mathrm{~ms}$. If the large-scale convection scenario discussed in Sect. 2.2 is possible, most of the differential rotation energy may be converted to magnetic energy by dynamo action. Let's first estimate the total differential rotation energy $E_{\mathrm{d}}$ in the case when the angular momentum of each mass element is conserved in the collapse,

$E_{\mathrm{d}}=\frac{16 \pi^{3} \rho}{3 P_{0}^{2}} \int_{0}^{R} r_{0}^{4} \mathrm{~d} r \sim 3.110^{46} P_{1}^{-2}$ erg. 
For a typical initial period $P=10 \mathrm{~ms}$, one gets $E_{\mathrm{d}} \sim$ $10^{50} \mathrm{erg}$. The dominant energy in the core-collapse supernovae is the gravitational energy $E_{\mathrm{g}} \sim 0.6 G \mathrm{M}^{2} / R \sim$ $310^{53} \mathrm{erg}$ for $M=1.4 M_{\odot}$ and $R=10^{6} \mathrm{~cm}$, if a strange star is residual. We thus find that a significant part ( $\sim 0.03 \%$ for $P=10 \mathrm{~ms}$ ) of the gravitational energy has to be converted to the rotation energy if angular momentum is conserved. If most of this differential rotation energy would be converted to magnetic energy by $\alpha-\Omega$ dynamo action, $\frac{B^{2}}{8 \pi} \sim \frac{E_{\mathrm{d}}-E_{\mathrm{f}}}{R^{3}}$, one obtains the saturation magnetic field in the interior of the stars,

$B_{\text {sat }} \sim 4.310^{14} P_{1}^{-1} \mathrm{G}$,

which is near the value estimated by Thompson \& Duncan (TD93) who assumed that most of the convective energy is converted to the magnetic one. $B_{\text {sat }} \sim 10^{16} \mathrm{G}$ for a typical period $P=10 \mathrm{~ms}$. Certainly, the actual "dipole" magnetic field $B_{\mathrm{p}}$ of pulsars should be only a fraction of $B_{\text {sat }}$. For example, in the upper convection zone of the sun, the rms value of the magnetic field is only $10^{-2} B_{\text {sat }}$. If the same is assumed for the pulsars, the rms value of fields in PSSs or PNSs should be $\sim 10^{12} P_{1}^{-1}$ G. However, the poloidal magnetic field should be another fraction of this rms value, thus

$B_{\mathrm{p}} \lesssim 10^{12} P_{1}^{-1} \quad \mathrm{G}$.

Observations for ordinary pulsars show that the fields are distributed from $\sim 10^{11} \mathrm{G}$ to $5.510^{13} \mathrm{G}$ (PSR J1814-1744). Therefore, it is likely the initial period $P$ of ordinary pulsars could be in the range of a few hundreds to decades of milliseconds. If $P<10 \mathrm{~ms}$, much stronger dipole fields (e.g., $\sim 10^{14}-10^{15} \mathrm{G}$ for magnetars) can be generated.

In the other scenario, where diffusivities based on neutrino scattering inhibit large-scale convection, the differential rotation is damped due to the momentum transport by neutrinos. In this case the dynamo may be driven dominantly by turbulent convection, and the field strength can be estimated as $B=\sqrt{4 \pi \rho v_{\mathrm{q}}^{2}} \sim 10^{13} \mathrm{G}$, assuming an equilibrium state between kinetic and magnetic energy. The efficiency of converting differential rotation energy into magnetic energy by dynamo action in this scenario may be much smaller than in the case where convection with the large scale $L$ exists since a significant fraction of differential rotation energy is likely to be converted into thermal energy due to the high neutrino viscosity.

Thus, dynamo action may amplify significantly the field before PSSs cools down to temperatures $T$ smaller than the critical one, $T_{\mathrm{c}}$. When $T<T_{\mathrm{c}}$, CSC appears, and the field would exist as a fossil one for a very long time since $\tau_{\mathrm{ohm}} \rightarrow \infty$ if $\sigma \rightarrow \infty$. In fact, Alford et al. (2000) investigated recently the effect of $\mathrm{CSC}$ on the magnetic fields, and found that, unlike the conventional superconductors where weak magnetic fields are expelled by the Meissner effect, color superconductors can be penetrated by external magnetic fields and such fields can exist stably on a timescale longer than the cosmic age.
Equation (39) may have an observational consequence for pulsar statistics, which in turn could test Eq. (39). In the magnetic dipole model of pulsars, $\left(3.210^{19}\right)^{2} P \frac{\mathrm{d} P}{\mathrm{~d} t}=$ $B_{\mathrm{p}}^{2}$ (note: fields are assumed not to decay in SQM), and the rotation period $P$ is a function of time $t$ due to energy loss. $P(0)$ denotes the initial period of pulsars. Considering Eq. (39), one has $P(t) \frac{\mathrm{d} P(t)}{\mathrm{d} t}=\zeta P(0)^{-2}$, the solution of which is

$P(t)^{2}=P(0)^{2}+2 \zeta P(0)^{-2} t$,

where $\zeta \sim 4.310^{-18} \mathrm{~s}^{3}$. Observational tests of this equation are highly desirable.

The condition $E_{\mathrm{d}}<E_{\mathrm{g}}$ gives a limit for the initial pulsar period $P$. Based on Eq. (37), one gets $P>0.2 \mathrm{~ms}$. Actually, $P \gg 0.2 \mathrm{~ms}$ since the efficiency of converting gravitation energy into differential rotation energy during collapse may be very small. It is thus doubtful that supernovae can produce pulsars with submilliseconds periods. For recycled millisecond pulsars the above estimate is not relevant.

\subsection{Fast dynamos}

The magnetic field amplification processes in newborn pulsars are essentially fast dynamos because of the high magnetic Reynolds numbers $\left(\mathcal{R}_{\mathrm{m}} \sim 10^{16}-10^{17}\right.$ for largescale convection, $\mathcal{R}_{\mathrm{m}} \sim 10^{10}$ for local turbulence) of both PSSs and PNSs. Unfortunately, the question of whether or not fast dynamo exist has not been answered theoretically although many numerical and analytical calculations strongly support the existence of kinematic fast dynamos for given sufficiently complicated flows (Soward 1994; Childress \& Gilbert 1995). It is worth studying fluids without magnetic diffusion since the diffusion timescale is much longer than the advection timescale, $\tau_{\mathrm{ohm}} \gg \tau_{\mathrm{v}}$, for fast dynamos. The complex flows, such as stretchtwist-fold, may effectively amplify the field in this case. The amplified strong magnetic fields are concentrated in filaments with radii $l_{\mathrm{f}}$, which can be estimated to be $l_{\mathrm{f}} \sim \mathcal{R}_{\mathrm{m}}^{-1 / 2} \check{L} \sim 0.01 / \sqrt{\mathcal{R}_{\mathrm{m} 16}} \mathrm{~mm}\left(\mathcal{R}_{\mathrm{m}}=\mathcal{R}_{\mathrm{m} 16} 10^{16}\right)$ by equating the diffusion timescale of filament field, $l_{\mathrm{f}}^{2} / \kappa_{\mathrm{m}}$, to the advection timescale $\tau_{\mathrm{v}}$. Fast dynamos in PNSs have been considered by Thompson \& Duncan (TD93) who suggested PNS dynamos as the origins of pulsar magnetism. As the fluid parameters (e.g., $\left.\mathcal{R}_{\mathrm{m}}, \nu, \kappa\right)$ of both PSSs and PNSs are similar, fast dynamos may also work for newborn strange stars.

There are three timescales in the fast dynamo of PSSs: the diffuse timescale $\tau_{\mathrm{ohm}}=\check{L}^{2} / \kappa_{\mathrm{m}}$, the advection timescale $\tau_{\mathrm{v}}=\check{L} / \check{U}$, and the buoyancy timescale $\tau_{\mathrm{b}}$,

$$
\begin{aligned}
\tau_{\mathrm{b}} & \approx 4 c \sqrt{\frac{\pi}{3 G M}} R L^{1 / 2} \rho^{1 / 2} B^{-1} \\
& \sim 10 \mathrm{~ms} R_{6} L_{5}^{1 / 2} \rho_{15}^{1 / 2} B_{16}^{-1}
\end{aligned}
$$

$\left(R=R_{6} \times 10^{6} \mathrm{~cm}, L=L_{5} \times 10^{5} \mathrm{~cm}, B=B_{16} 10^{16} \mathrm{G}\right)$. $B_{16} \sim 1.12 \rho_{15}^{1 / 2} v_{8}\left(v=v_{8} \times 10^{8} \mathrm{~cm} \mathrm{~s}^{-1}\right)$ if we assume 
that kinetic and magnetic energy densities are in equilibrium. Filaments can be generated inside the convective layer since turbulent convection is violent on small scales $(\sim 100 \mathrm{~cm}) . \tau_{\mathrm{v}} \lesssim \tau_{\mathrm{b}} \ll \tau_{\mathrm{ohm}}$, which means that filaments rise to the stellar surface by magnetic buoyancy as soon as strong fields are created by fast dynamos. Magnetic buoyancy flow or convection could create and amplify the poloidal (transverse) field from the azimuthal (aligned) field, at the same time, the aligned field could also be created and amplified from the transverse field by differential rotation as discussed in Sect. 2.1. Thus these processes may complete a "dynamo cycle" in newborn pulsars.

Let's give some estimates for fast dynamos. There are mainly two types of flows in PSSs: eddy (convection) and shear (differential rotation). Actually, these flows are coupled. However, we may deal with them separately in order to have an overview of the field generation processes. For pure straining motion with velocity field $\mathcal{V}$,

$\mathcal{V}=\tau_{\mathrm{v}}^{-1}(-x, y, 0)$,

which represents eddy flow to some extent, the magnetic field can be amplified considerably to $B_{\max }^{\mathrm{e}}=B_{0} \mathcal{R}_{\mathrm{m}}^{1 / 2}$ at a timescale $t_{\max }^{\mathrm{e}}=\frac{1}{2} \tau_{\mathrm{v}} \ln \mathcal{R}_{\mathrm{m}}^{1 / 2}$ (Soward 1994). For an initial field strength $B_{0} \sim 10^{10} \mathrm{G}, B_{\max }^{\mathrm{e}} \sim 10^{18} \mathcal{R}_{\mathrm{m} 16}^{1 / 2} \mathrm{G}$ and $t_{\max }^{\mathrm{e}} \sim 18 \tau_{\mathrm{v} 3} \ln \mathcal{R}_{\mathrm{m} 16}^{1 / 2} \operatorname{ms}\left(\tau_{\mathrm{v}}=\tau_{\mathrm{v} 3} \times 10^{-3} \mathrm{~s}\right)$. On another hand, the linear shear flow (Soward 1994),

$\mathcal{V}=T_{\mathrm{v}}^{-1}(y, 0,0)$,

can amplify the field to $B_{\max }^{\mathrm{s}} \sim B_{0} \mathcal{R}_{\mathrm{m}}^{1 / 3} \sim 210^{15} \mathcal{R}_{\mathrm{m} 16}^{1 / 3}$ $\mathrm{G}$ at a growth time $t_{\max }^{\mathrm{s}} \sim T_{\mathrm{v}} \mathcal{R}_{\mathrm{m}}^{1 / 3} \sim 21 T_{\mathrm{v} 4} \mathcal{R}_{\mathrm{m} 16}^{1 / 3} \mathrm{~s}$ for $B_{0}=10^{10} \mathrm{G}\left(T_{\mathrm{v}}=10^{-4} T_{\mathrm{v} 4} \sim L /(V(R)-V(R-L))\right.$, see Fig. 2). The timescale for field generation is thus from $10^{-5} \mathrm{~s}$ (for $\mathcal{R}_{\mathrm{m}} \sim 10^{10}$ ) to $10 \mathrm{~s}$ (for $\mathcal{R}_{\mathrm{m}} \sim 10^{16}$ ), while the amplified fields could be $10^{12}$ (for $\mathcal{R}_{\mathrm{m}} \sim 10^{10}$ ) to $10^{18}$ (for $\mathcal{R}_{\mathrm{m}} \sim 10^{16}$ ) $\mathrm{G}$ in filaments. The magnetic fields emerging from under the stellar surface are likely to be much smaller than $B_{\max }$ in filaments because the filaments will expand to an approximately homogeneous field near and above the surface owing to magnetic pressure. Incoherent or coherent superposition of this buoyant field flux might result in the observed dipole moment of pulsars (TD93).

Differential rotation may play an essential role for the generation of large-scale magnetic fields through $\alpha-\Omega$ dynamo process, but even in the absence of differential rotation, large-scale magnetic fields may be created. Owing to the alignment of small-scale convection rolls parallel to the axis of rotation, global magnetic fields can be generated as has been shown in various dynamo models (see, for instance, Busse 1975).

\section{Any differences of dynamo-originated fields?}

As discussed in the previous section, magnetic dipole fields stronger than $10^{11} \mathrm{G}$ could be generated by dynamos in PSSs. However, if pulsars are born as strange stars, an acute question is how to explain the fields of millisecond pulsars with $\sim 10^{8} \mathrm{G}$ since fields don't decay in strange stars with CSC. Millisecond pulsars are supposed to be spun-up ("recycled") as the result of accretion from companion stars in their histories. They could be strange stars with $\sim 10^{-5} M_{\odot}$ crusts, although a lot of ordinary pulsars might be strange stars with bare polar caps and much thinner crusts (Xu et al. 2001). We suggest that accretion may reduce the dipole field strength from $\sim 10^{12} \mathrm{G}$ to $\sim 10^{8} \mathrm{G}$ of strange stars in four possible ways: (1) the accreted matter may screen or bury the original fields (Bisnovati-Kogan \& Komberg 1974; Zhang et al. 1994; Zhang 2000); (2) field decays in the accretionheated crust (Konar \& Bhattacharya 1997); (3) the large scale fields (e.g., dipole structure) could be changed significantly because the plasma accreted onto the polar caps would squeeze the frozen magnetic fields toward the equator (Cheng \& Zhang 1998), and the dipole field thus appears to decay; (4) magnetic fields frozen in the bottom crust may dissipate and be annihilated during the combustion process of the bottom matter into SQM when the crust becomes too heavy to be supported by the Coulomb barrier.

A notable difference between strange stars and neutron stars is that the magnetic field of a strange core is stable and does not decay due to CSC, while the field of a neutron star should be expelled from the interior to the crust where the Ohmic dissipation occurs. In present models for neutron star magnetism, the fields permeate either the whole star or only the crust (see, e.g., a short review by Mitra et al. 1999). It is found by observational and statistical analyses that a pulsar's field can only decrease substantially in the accretion-phase but does not decay significantly during the pulsar lifetime (Hartman et al. 1997). New calculations of the timescale for Ohmic dissipation in the crust have shown that the field can persist for more than $10^{10}$ years (Sang \& Chanmugam 1987). It thus may be difficult to distinguish the field evolutions of strange stars and neutron stars in the radio pulsar-phase. Nevertheless, the field decay modes in the accretion-phase could be quite different for neutron stars and strange stars. In fact the items (1)-(3) in the above proposals for field decay are relevant to both neutron stars and strange stars, but item (4) can only apply to strange stars. Furthermore, items (1)-(3) could result in different physical processes for strange star and neutron star because they have very different structures. For example, as matter accretes, the radius of a strange star increases, while the radius of a neutron star decreases. Also, the material in neutron star crust moves continuously, and the movement just pushes and squeezes the original field. However for strange stars, a SQM phase-transition occurs when the accreted material moves across the Coulomb barrier with thickness of $\sim 200 \mathrm{fm}$. Actually, this phasetransition process has been included in the explanation of some burst phenomena, such as bursting X-ray pulsar GRO J1744-28 (Cheng et al. 1998), but the field evolution in accreting strange star has not yet been discussed extensively. We propose here that strange stars and neutron stars may be distinguished by their field decays during the 
accretion-phases. Future investigations of this issue will be of interest.

As addressed in the previous sections, many fluid parameters for the large-scale convection scenario in PSSs and PNSs are similar. Both kind of stars have convective layers with thickness $\sim 10^{5} \mathrm{~cm}$ and flow velocities $\sim 10^{8} \mathrm{~cm} \mathrm{~s}^{-1}$. The scale length of turbulent eddies of both $\mathrm{PSS}$ and PNS is $\sim 2 \mathrm{~m}$ since neutrino viscosity damps flow only on scale greater than $l \sim l^{\mathrm{N}} \sim 2 \mathrm{~m}$. The neutrino viscosities and the magnetic Reynolds numbers in PSSs and PNSs are also not quite different: $\nu \sim 10^{10} \mathrm{~cm}^{2} / \mathrm{s}, \nu^{\mathrm{N}} \sim$ $10^{8} \mathrm{~cm}^{2} / \mathrm{s} ; \mathcal{R}_{\mathrm{m}} \sim 10^{16}, \mathcal{R}_{\mathrm{m}}^{\mathrm{N}} \sim 10^{17}$. Therefore, the general configurations and strength of dynamo-generated magnetic fields in PSSs and PNSs may be similar. However, the fluid properties calculated in this paper for SQM are rather uncertain since our knowledge of SQM fluid is less than that of nuclear matter. Detailed studies of the fluid properties in PSSs, such as the neutrino fraction and viscosity, are necessary and may help us to see differences in the dynamo-originated fields. In addition, the timescale available for dynamo action in PSSs may be significantly smaller than that in PNSs since the energy gap for proton superconductivity is order of $1 \mathrm{MeV}$ (rather than 10$100 \mathrm{MeV}$ in SQM). This property may also affect the structure of the fields.

\section{Conclusion and discussion}

Pulsars could be neutron stars and/or strange stars. The origin of neutron star magnetic fields has been discussed in literature, while few papers are concerned with the origin of fields in strange stars. In this paper, we have investigated the dynamo action in PSSs, and have suggested that strange stars can have magnetic fields of dynamo origin similar to those in neutron stars. Our main conclusions are as follows.

1. A significant fraction of gravitational energy has to be converted to differential rotation energy if the angular momentum of each mass element is conserved in the core collapse. Assuming an initial rotating core to be approximated by the model of Bruenn (1985), we have calculated the rotation velocity, the velocity derivative, and the differential rotation energy of a nascent strange star.

2. It is found that PSSs may have a convective layer of thickness $\sim 2 \mathrm{~km}$ in the outer part. The large-scale convection has a velocity $\sim 10^{8} \mathrm{~cm} \mathrm{~s}^{-1}$, while local turbulent eddies have a scale of $\sim 1 \mathrm{~m}$ and a velocity of $\sim 10^{5} \mathrm{~cm} \mathrm{~s}^{-1}$.

3 . The energy density of differential rotation could be larger than the turbulent energy density if the pulsar initial period $P \lesssim 10 \mathrm{~ms}$. Assuming that most of the differential rotation energy is converted to magnetic energy by dynamo action, we obtain the dipole field strength as a function of $P$ and the pulsar period evolution due to magnetic dipole radiation.

4. The fields $\left(10^{12}-10^{18} \mathrm{G}\right)$ amplified by fast dynamo action are concentrated in filaments with initial radii $\sim 0.01 \mathrm{~mm}-\sim 1 \mathrm{~cm}$ and with growth times $10^{-5} \mathrm{~s}$ to $10 \mathrm{~s}$.
5. Strange stars and neutron stars are expected to have different accretion-induced field decay processes, which could be used to distinguish them in the future.

6. Convection with the large scale $L$ is less likely to exist in PSSs than in PNSs.

It is currently believed that anomalous X-ray pulsars (AXPs) and soft gamma repeaters (SGRs) are magnetismpowered in the "magnetar" model (Duncan \& Thompson 1992; Thompson \& Duncan 1995). The magnetic reconnection near the surface causes the emission in AXP and SGR. Duncan \& Thompson (1992, TD93) suggested that the key parameter that determines whether a PNS becomes an ordinary pulsar or a magnetar is the Rossby number. If magnetars are strange stars, we propose that, besides this key parameter, the initial temperature and the density of trapped neutrinos can also affect the formation of a magnetar since dynamo action could not be very effective when CSC appears. Strange stars with very strong magnetic field may act as "magnetars" since they could have similar differentiated structure as neutron stars (Benvenuto et al. 1990). However the neutron magnetar model faces a crisis indicated by Pérez Martinez et al. (2000) in that neutron stars may undergo a transverse collapse if their fields exceed a critical value. But for strange magnetars, a relatively small magnetic momentum and large chemical potential of free quarks may favor the formation of very high fields, although a further investigation is needed to find the critical field strength beyond which a strange star cannot be sustained against transverse collapse.

This paper may have two implications for the studies of the $r$-mode instability (Anderson 1998; Madsen 1998, 2000; Lindblom et al. 1998). (1) As discussed in Sect. 2.1, PSSs and PNSs should rotate strongly differentially (particularly for $P \lesssim 10 \mathrm{~ms}$ ). But the numerical studies that have appeared in the literature are for stars with uniform rotation. Certainly, it is reasonable to assume that the result for uniform rotation is qualitatively representative also for the case of differential rotation. (2) The dynamo-originated strong magnetic fields inside strange stars or neutron stars should be included in investigations of the $r$-mode instabilities in pulsars. Although a very short period $P$ will be favourable for the occurrence of $r$-mode instability, a smaller $P$ would also be favourable for the generation of a stronger magnetic field by dynamo action, thus exerting a stabilizing influence. As the temperature decreases, the bulk viscosity also decreases and the $r$-mode instability may occur if there are no other strong dissipative effects. Previously, Rezzolla et al. (2000) have shown that the interaction of $r$-mode oscillations with the magnetic field could be important and that the oscillations will be inhibited if the field is of the order $\sim 10^{16}\left(\Omega / \Omega_{\mathrm{B}}\right) \mathrm{G}$, where $\Omega$ is the angular velocity of the star and $\Omega_{\mathrm{B}}$ is the value at which mass shedding occurs. Since the fields of PSSs (or PNSs) depend on the nature of turbulence and on the rotation frequency, further study is needed to see whether dynamo-generated fields can affect significantly the $r$-mode oscillation. 
Acknowledgements. This research was supported by National Nature Sciences Foundation of China (19803001), and by the Special Funds for Major State Basic Research Projects of China. We thank an anonymous referee for helpful suggestions.

\section{References}

Alcock, C., Farhi, E., \& Olinto, A. 1986, ApJ, 310, 261

Alford, M., Rajagopal, K., \& Wilczek, K. 1999, Nucl. Phys. B, 537,443

Alford, M., Berges, J., \& Rajagopal, K. 2000, Nucl. Phys. B, 571,269

Anand, J. D., Goyal, A., Gupta, V. K., \& Singh, S. 1997, ApJ, 481,954

Anderson, N. 1998, ApJ, 502, 708

Arnett, W. D. 1977, ApJ, 218, 815

Benvenuto, O. G., \& Horvath, J. E. 1989, Phys. Rev. Lett., 63, 716

Benvenuto, O. G., Horvath, J. E., \& Vucetich, H. 1989, Int. J. Mod. Phys. A, 4, 257

Benvenuto, O. G., Horvath, J. E., \& Vucetich, H. 1990, Phys. Rev. Lett., 64, 713

Benvenuto, O. G., \& Lugones, G. 1999, MNRAS, 304, L25

Bisnovati-Kogan, G., \& Komberg, B. 1974, Sov. Astron., 18, 217

Bodmer, A. R. 1971, Phys. Rev. D, 4, 1601

Böhm-Vitense, E. 1958, Z. Astrophys., 46, 108

Bruenn, S. W. 1985, ApJSS, 58, 771

Burrows, A., \& Lattimer, J. M. 1988, Phys. Rep., 163, 51

Busse, F. H. 1975, Geophys. J. R. Astr. Soc., 42, 437

Chamaj, T., \& Slominski, W. 1989, Phys. Rev. D, 40, 165

Cheng, K. S., Dai, Z. G., Wei, D. M., \& Lu, T. 1998, Science, 280,407

Cheng, K. S., \& Zhang, C. M. 1998, A\&A, 337, 441

Childress, S., \& Gilbert, A. D. 1995, Stretch, Twist, Fold: The Fast Dynamo (Springer-Verlag, Berlin)

Cleymans, R., Gavay, V., \& Suhonen, E. 1986, Phys. Rep., 130, 219

Clever, R. M., \& Busse, F. H. 1981, J. Fluid Mech., 102, 61

Dai, Z. G., Peng, Q. H., \& Lu, T. 1995, ApJ, 440, 815

Drago, A. 1999, in Neutron stars, Supernova Explosions and the Transition to Quark Matter, 19th Texas Symposium on Relativistic Astrophysics and Cosmology, ed. J. Paul, T. Montmerle, \& E. Aubourgthe, Paris, 217

Duncan, R. C., \& Thompson, C. 1992 ApJ, 392, L9

Glendenning, N. K. 1992, Phys. Rev. D, 46, 1274

Goussard, J.-O., Haensel, P., \& Zudunik, J. L. 1998, A\&A, 344, 117

Hartman, J. W., Bhattacharya, D., Wijers, R., \& Verbunt, F. 1997, A\&A, 322, 477

Heiselberg, H., \& Hjorth-Jensen, M. 2000, Phys. Rep., 328, 237

Heiselberg, H., \& Pethick, C. J. 1993, Phys. Rev. D, 48, 2916
Horvath, J. E., \& Benvenuto, O. G. 1988, Phys. Lett. B, 213, 516

Iwamoto, N. 1982, Ann. Phys., 141, 1

Janka, H.-T., \& Mönchmeyer, R. 1989, A\&A, 226, 69

Keil, W., \& Janka, H.-T. 1995, A\&A, 296, 145

Konar, S., \& Bhattacharya, D. 1997, MNRAS, 284, 311

Kouveliotou, C., et al. 1998, Nature, 393, 235

Lai, D., \& Shapiro, S. L. 1991, ApJ, 383, 745

Li, X.-D., Bombaci, I., Dey, M., Dey, J., \& van den Heuvel, E. P. J. 1999, Phys. Rev. Lett., 83, 3776

Lindblom, L., Owen, B. J., \& Morsink, S. M. 1998, Phys. Rev. Lett., 4843

Lugones, G., \& Benvenuto, O. G. 1998, Phys. Rev. D, 58, 083001

Lugones, G., Benvenuto, O. G., \& Vucetich, H. 1994, Phys. Rev. D, 50, 6100

Madsen, J. 1992, Phys. Rev. D, 46, 3290

Madsen, J. 1998, Phys. Rev. Lett., 81, 3311

Madsen, J. 1999, in Physics and Astrophysics of Strange Quark Matter, Hadrons in Dense Matter and Hadrosynthesis, ed. J. Cleymans, H. B. Geyer, \& F. G. Scholtz (Springer, Berlin), 162

Madsen, J. 2000, Phys. Rev. Lett., 85, 10

Malkus, W. V. R. 1954, Proc. Roy. Soc. A, 225, 196

Miralles, J. A., Pons, J. A., \& Urpin, V. 2000, ApJ, 543, 1001

Mitra, D., Konar, S., \& Bhattacharya, D. 1999, MNRAS, 307, 459

Moffatt, H. K. 1978, Magnetic Field Generation in Electrically Conducting Fluids (Cambridge Univ. Press, Cambridge)

Olinto, A. V. 1987, Phys. Lett. B, 192, 71

Pérez Martinez, A., Pérez Rojas, H., \& Mosquera Cuesta, H. J. 2000, submitted [hep-ph/0011399]

Rezzolla, L., Lamb, F. K., \& Shapiro, S. L. 2000, ApJ, 531, L139

Sang, Y., \& Chanmugam, G. 1987, ApJ, 323, L61

Soward, A. M. 1994, in Fast Dynamos, ed. M. R. E. Proctor, \& A. D. Gilbert, Lectures on Solar and Planetary Dynamos (Cambridge Univ. Press, Cambridge), 181

Spruit, H., \& Phinney, E. S. 1998, Nature, 393, 139

Tatsumi, T. 2000 [astro-ph/0004062]

Thompson, C., \& Duncan, R. C. 1993, ApJ, 408, 194 (TD93)

Thompson, C., \& Duncan, R. C. 1995, MNRAS, 275, 255

Usov, V. V. 1998, Phys. Rev. Lett., 81, 4775

van Atta, C. 1966, J. Fluid Mech., 25, 495

Wang, Q. D., \& Lu, T. 1984, Phys. Lett. B, 148, 211

Wilson, J. R., \& Mayle, R. W. 1988, Phys. Rep., 163, 63

Witten, E. 1984, Phys. Rev. D, 30, 272

Xu, R. X., Qiao, G. J., \& Zhang, B. 1999, ApJ, 522, L109

Xu, R. X., Zhang, B., \& Qiao, G. J. 2001, Astroparticle Phys., 15, 101 [astro-ph/0006021]

Zhang, C. M. 2000, Int. J. Mod. Phys. D, 9, 1

Zhang, C. M., Wu, X. J., \& Yang, G. C. 1994, A\&A, 283, 889

Zhang, K. 1995, J. Fluid Mech., 284, 239 